\title{
CD70 Expression and Its Correlation with Clinicopathological Variables in Squamous Cell Carcinoma of the Head and Neck
}

\author{
Astrid De Meulenaere $^{b} \quad$ Tijl Vermassen $^{b} \quad$ Sandrine Aspeslagh $^{f}$ \\ Karen Zwaenepoel ${ }^{\mathrm{a}}$ Philippe Deron $^{\mathrm{c}}$ Fréderic Duprez ${ }^{\mathrm{d}}$ Liesbeth Ferdinande ${ }^{\mathrm{e}}$ \\ Sylvie Rottey ${ }^{b}$ \\ ${ }^{\mathrm{a}}$ Department of Pathology, Antwerp University Hospital, Edegem, and Departments of ${ }^{\mathrm{b}}$ Medical Oncology, \\ ${ }^{c}$ Head, Neck and Maxillo-Facial Surgery, ${ }^{d}$ Radiation Oncology and e Pathology, Ghent University Hospital,

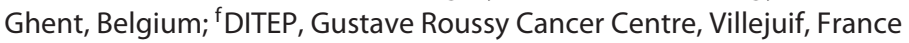

\section{Key Words}

Squamous cell carcinoma of the head and neck - CD70 .

Tumor-infiltrating lymphocytes · Differentiation grade

\begin{abstract}
Objective: Over the last decade, efforts have been made to get a better understanding of the tumor microenvironment and the role of the immune system in it. New insights into the CD27/CD70 signaling pathway point towards a role in tumor immunology, making CD70 an attractive target for immunotherapy. Here, we evaluate CD70 expression in squamous cell carcinoma of the head and neck (SCCHN). Methods: CD70 immunohistochemistry was retrospectively performed on 95 tumor samples. Tumoral CD70 expression was scored and correlated with clinicopathological variables and overall survival (OS). Results: CD70 expression in tumor cells was observed in 66 samples (69\%) and was strongly associated with tumor differentiation grade $(p<0.001)$. CD70 expression was also observed in tumor-associated fibroblasts and endothelial cells. Additionally, the density of tumor-infiltrating lymphocytes correlated with OS $(p=0.042)$.
\end{abstract}

\section{KARGER}

E-Mail karger@karger.com

www.karger.com/pat
Conclusion: This study describes the tumoral expression of CD70 in SCCHN. Results highlight the role of CD70 in tumor biology and identify CD70 as a novel therapeutic target. Further research is warranted.

(c) 2016 S. Karger AG, Basel

\section{Introduction}

Squamous cell carcinoma of the head and neck (SCCHN), the sixth most common malignancy worldwide [1], is currently treated by a combination of surgery, radiation and chemotherapy, depending on the cancer staging $[2,3]$. Because survival rates for SCCHN have remained relatively stagnant over the last decades [2], there is an urgent need for new therapeutic agents that act in a more selective/effective and less toxic/aggressive manner to allow safe and personalized therapy. Hitherto, targeted therapy in SCCHN is confined to anti-EGFR therapy (in combination with conventional chemotherapies and radiotherapy) [4]. Unfortunately, primary resistance against anti-EGFR therapeutics is very high and ultimate-
(C) 2016 S. Karger AG, Basel

$1015-2008 / 16 / 0836-0327 \$ 39.50 / 0$ 
Table 1. Patient and tumor characteristics

\begin{tabular}{|c|c|c|c|c|c|}
\hline & $\begin{array}{l}\text { Hypopharynx } \\
(\mathrm{n}=15)\end{array}$ & $\begin{array}{l}\text { Larynx } \\
(n=23)\end{array}$ & $\begin{array}{l}\text { Oral cavity } \\
(\mathrm{n}=25)\end{array}$ & $\begin{array}{l}\text { Oropharynx } \\
(\mathrm{n}=32)\end{array}$ & $\begin{array}{l}\text { Total } \\
(\mathrm{n}=95)\end{array}$ \\
\hline \multicolumn{6}{|l|}{ Age, years } \\
\hline $15-45$ & $1(7)$ & $0(0)$ & $1(4)$ & $2(6)$ & $4(4)$ \\
\hline $46-74$ & $14(93)$ & $21(91)$ & $19(76)$ & $20(63)$ & $74(78)$ \\
\hline$\geq 75$ & $0(0)$ & $2(9)$ & $5(20)$ & $10(31)$ & $17(18)$ \\
\hline \multicolumn{6}{|l|}{ Gender } \\
\hline Male & $14(93)$ & $23(100)$ & $15(60)$ & $21(66)$ & $73(77)$ \\
\hline Female & $1(7)$ & $0(0)$ & $10(40)$ & $11(34)$ & $22(23)$ \\
\hline \multicolumn{6}{|l|}{ T-staging } \\
\hline $\mathrm{T} 1-\mathrm{T} 2$ & $6(40)$ & $12(52)$ & $20(80)$ & $19(59)$ & $57(60)$ \\
\hline T3-T4 & $9(60)$ & $11(48)$ & $5(20)$ & $13(41)$ & $38(40)$ \\
\hline \multicolumn{6}{|l|}{ N-staging } \\
\hline N0-N1 & $12(80)$ & $19(83)$ & $22(88)$ & $15(47)$ & $68(72)$ \\
\hline $\mathrm{N} 2 \mathrm{a}-\mathrm{N} 2 \mathrm{~b}$ & $1(7)$ & $1(4)$ & $1(4)$ & $9(28)$ & $12(13)$ \\
\hline $\mathrm{N} 2 \mathrm{c}-\mathrm{N} 3$ & $2(13)$ & $3(13)$ & $2(8)$ & $8(25)$ & $15(16)$ \\
\hline \multicolumn{6}{|l|}{ M-staging } \\
\hline Mo & $15(100)$ & $23(100)$ & $25(100)$ & $30(94)$ & $93(98)$ \\
\hline M1 & $0(0)$ & $0(0)$ & $0(0)$ & $2(6)$ & $2(2)$ \\
\hline \multicolumn{6}{|l|}{ HPV status } \\
\hline Positive & $0(0)$ & $1(4)$ & $0(0)$ & $4(13)$ & $5(5)$ \\
\hline Negative & $15(100)$ & $22(96)$ & $25(100)$ & $28(88)$ & $90(95)$ \\
\hline \multicolumn{6}{|l|}{ Smoking history } \\
\hline Positive & $15(100)$ & $23(100)$ & $21(84)$ & $27(84)$ & $86(91)$ \\
\hline Negative & $0(0)$ & $0(0)$ & $4(16)$ & $5(16)$ & $9(9)$ \\
\hline Median OS, days & 327 & 1.021 & 826 & 806 & \\
\hline
\end{tabular}

ly all patients will develop secondary resistance [5]. Preliminary results of anti PD-1 therapy [6] reflected the importance of the immune system in the fight against head and neck cancer, inciting us to further analyze other immunomodulatory pathways.

The CD27/CD70 costimulatory pathway is part of the TNF receptor/ligand family and regulates cellular activity in subsets of T, B and natural killer cells [7]. Signaling through the CD27 receptor is limited, predominantly because of the restricted expression pattern of its ligand CD70; CD70 is only transiently expressed on T and B cells following antigen activation and on mature dendritic cells [7]. However, CD70 expression has been reported in a variety of human malignancies (e.g. lymphoma, leukemia, renal cell carcinoma and nasopharyngeal carcinoma) [8]. In this context, CD70 contributes to an immunosuppressive microenvironment by augmenting and/or attracting regulatory $\mathrm{T}$ cells, inducing $\mathrm{T}$ cell apoptosis and stimulating $\mathrm{T}$ cell exhaustion [9-11]. These findings render CD70 an attractive target for cancer therapy. First, blocking CD70 would enhance antitumor immunity by reducing its immunosuppressive impact on tumor-infiltrating lymphocytes (TILs). Second, via a direct mode of action, anti-CD70 antibodies would induce antibody-dependent cellular cytotoxicity in CD70-expressing cells [12]. To date, CD70 expression has not been investigated in SCCHN. The purpose of this study was to describe the expression pattern of CD70 in SCCHN by immunohistochemistry (IHC) and correlate it with clinicopathological variables.

\section{Materials and Methods}

\section{Patient and Tissue Selection}

Patients with histologically proven SCCHN in 2007-2012 were selected from the archival database of the Department of Pathology, Ghent University Hospital. Selection was based on the primary tumor site, the TNM stage and the availability of paraffinembedded tumor tissue to obtain a mixed set of SCCHN tumor samples representative of this diversified group of tumors. A total 

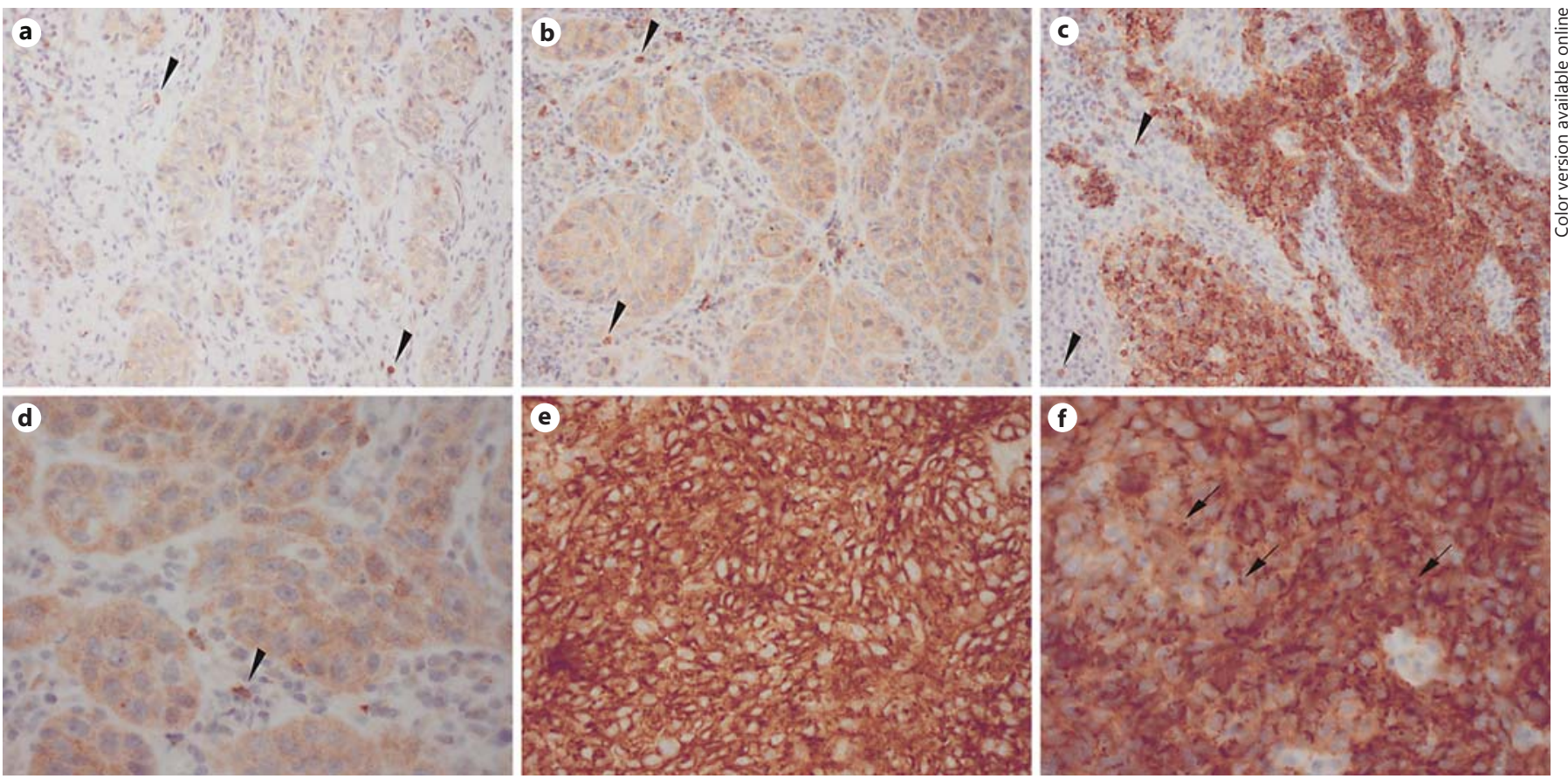

Fig. 1. Representative CD70 IHC. a-c CD70 staining intensity score: weak (a), moderate (b) and strong (c). $\times 200$. d-f CD70 staining pattern on tumor cells was cytoplasmatic (d), membranous (e) and dot-like (f, arrows). $\times 400$. Note the strong cytoplasmatic staining in TILs (arrowheads), used as an internal positive staining control.

of 95 primary tumor samples from 95 patients were included in this study. When available, samples from resection specimens were preferred for immunohistochemical analysis $(\mathrm{n}=36)$; otherwise, endoscopic biopsy material was used $(n=59)$. The study was approved by the ethics committee of the Ghent University Hospital.

\section{Patient and Tumor Characteristics}

The study cohort consisted of 95 patients, all diagnosed with SCCHN. This included 15 cases of hypopharyngeal cancer, 23 of laryngeal cancer, 25 of cancer of the oral cavity and 32 of oropharyngeal cancer. The patient and tumor characteristics are presented in table 1.

\section{IHC}

Formalin-fixed paraffin-embedded tissue slides of $2 \mu \mathrm{m}$ were used for IHC. Heat-induced antigen retrieval was performed with a high $\mathrm{pH}$ buffer $\left(20 \mathrm{~min}\right.$ at $\left.97^{\circ} \mathrm{C}\right)$. The following steps were performed in an Autostainer Link 48 instrument (Dako, Glostrup, Denmark): 5 min of incubation in peroxidase-blocking buffer followed by 20 min of incubation with anti-CD70 primary antibody (clone 301731 diluted 1:40; R\&D Systems Inc., Minneapolis, Md., USA) and DAB detection using the EnVision FLEX+ kit (Dako) according to the instructions of the manufacturer. Slides were counterstained with hematoxylin.

\section{Microscopic Evaluation of Tumor Samples}

First, a hematoxylin-eosin slide was evaluated to confirm the presence of invasive squamous cell carcinoma and to determine the grade of tumor differentiation. The infiltration of TILs in tumor stroma was assessed semiquantitatively as minimal (+), low $(++)$, intermediate $(+++)$ or high $(++++)$ infiltration of TILs. Subsequently, CD70 IHC was scored for the proportion (percentage of tumor cells) and intensity (weak, moderate or strong) of CD70 staining in tumor cells (fig. 1a-c). Different types of staining patterns in the tumor were documented, namely, cytoplasmic, membranous, dot-like and, very seldom, nuclear (fig. 1d-f).

\section{Statistical Analysis}

Statistical analysis was performed using SPSS v22.0 (IBM Corp., Armonk, N.Y., USA) and GraphPad Prism 4.03 (GraphPad Software Inc., La Jolla, Calif., USA) to evaluate the relation between tumoral CD70 expression (continuous variable) and different clinicopathological (categorical) variables, such as primary tumor site, grade of differentiation, TILs and TNM stage.

Normal distribution was verified by the D'Agostino-Pearson test. Differences in CD70 expression between primary tumor site, grade of differentiation, TILs, and, TNM stage were determined by means of Kruskal-Wallis test. Intergroup differences were further analyzed by means of Dunn's Multiple Comparison test. Next, the influence of CD70 expression on overall survival (OS) in the cohort was determined by the Kaplan-Meier analysis. To assess the association between CD70 expression and OS, we dichotomized the continuous CD70 scores into 'positive' and 'negative' expression (10\% cut-off) [13]. Lastly, we determined the effect of TILs on OS. $p<0.05$ was considered statistically significant. 
Fig. 2. Expression of CD70 expression on endothelial cells and fibroblasts. a Weak cytoplasmatic staining of endothelial cells (lining the vessels indicated by the arrows). $\times 200$. b Moderate-to-strong cytoplasmatic staining of fibroblasts in desmoplastic stroma of an otherwise CD70-negative SCCHN. $\times 200$.
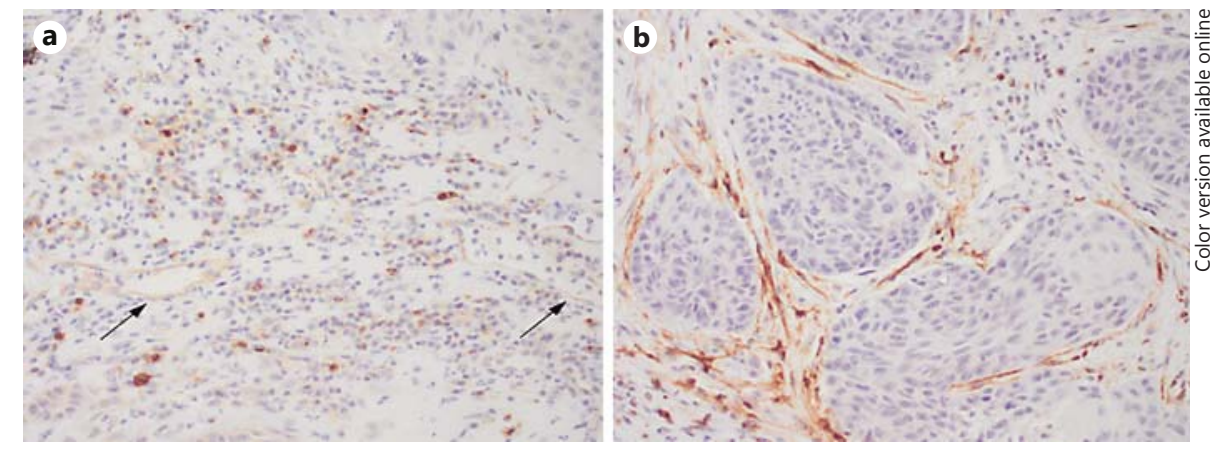

\section{Results}

\section{CD70 Expression in the Tumor}

CD70 expression in tumor cells was observed in 66/95 (69\%) samples with proportion scores ranging from 1 to $100 \%$. Forty-five of 66 cases $(68 \%)$ expressed CD70 on $\leq 10 \%$ of the tumor cells. Only 5 cases $(8 \%)$ were reported where $>90 \%$ of the tumor cells expressed CD70. The remaining samples stained for between 10 and 90\%.

Membranous staining was seen in 14 CD70-positive samples $(21 \%)$. Samples with a strong staining intensity for CD70, in particular, also had a membranous staining.

\section{CD70 Expression in the Tumor Microenvironment}

Besides its variable expression in the tumor cells, we analyzed CD70 expression on other cell types as well. A subset of TILs showed strong expression of CD70, in such a way that this expression pattern could be used as an internal positive control for staining quality. Occasionally, weak-to-moderate CD70 staining was observed in endothelial cells (fig. 2a). In addition, specifically in cases with extensive desmoplastic stromal reaction, weak-to-moderate CD70 positivity was seen in tumor-associated fibroblasts (fig. 2b).

\section{Correlation of CD70 Expression with \\ Clinicopathological Variables}

CD70 expression was strongly associated with the grade of differentiation ( $p<0.001$; fig. 3a), i.e. CD70 expression was significantly higher in poorly differentiated squamous cell carcinomas compared to moderately and well-differentiated carcinomas ( $\mathrm{p}<0.05$ and $\mathrm{p}<0.001$, respectively).

No significant association was found between CD70 expression and TNM stage ( $\mathrm{p}=0.433$; fig. $3 \mathrm{~b}$ ).

Although differences in primary tumor site did not reach statistical significance, CD70 expression appeared to be higher in patients with an oropharyngeal malignancy $(\mathrm{p}=0.064$; fig. $3 \mathrm{c})$.

Interestingly, we observed a trend that a high level of CD70 expression was associated with a lower density of TILs (fig. 3d). In addition, we noticed a significant association between the density of TILs and OS ( $p=0.042$; fig. 4): patients with tumors highly infiltrated by $\mathrm{T}$ cells had an improved outcome compared to patients with a low number of TILs.

There was no significant association between CD70 expression and OS ( $\mathrm{p}=0.681$; fig. 5).

\section{Discussion}

In this study, we investigated the immunohistochemical expression pattern of CD70 in a series of SCCHN $(\mathrm{n}=95)$. Sixty-nine percent of the samples showed a positive reactivity, ranging from 1 to $100 \%$ of tumor cells. CD70 expression was strongly associated with the grade of differentiation: a high CD70 proportion score was associated with a loss of differentiation. It seems that, especially in poorly differentiated SCCHN, CD70 is implicated in the process of immune escape, thereby promoting cancer progression [9-11].

As 69\% (66/95) of the SCCHN were found to express CD70, CD70-targeted immunotherapy might represent a new therapeutic option for this patient population. Currently, two anti-CD70 antibodies (SGN-CD70A and ARGX-110) are being explored in clinical trials. Although CD70 positivity of the tumor is a prerequisite to enter these trials, the use of CD70 IHC as a predictive marker to indicate treatment outcome is still under investigation. Hitherto, there is no uniform reporting of CD70 IHC in malignancies in the literature. The use of different methods (formalin-fixed paraffin-embedded or frozen-section), different antibody clones and different thresholds 


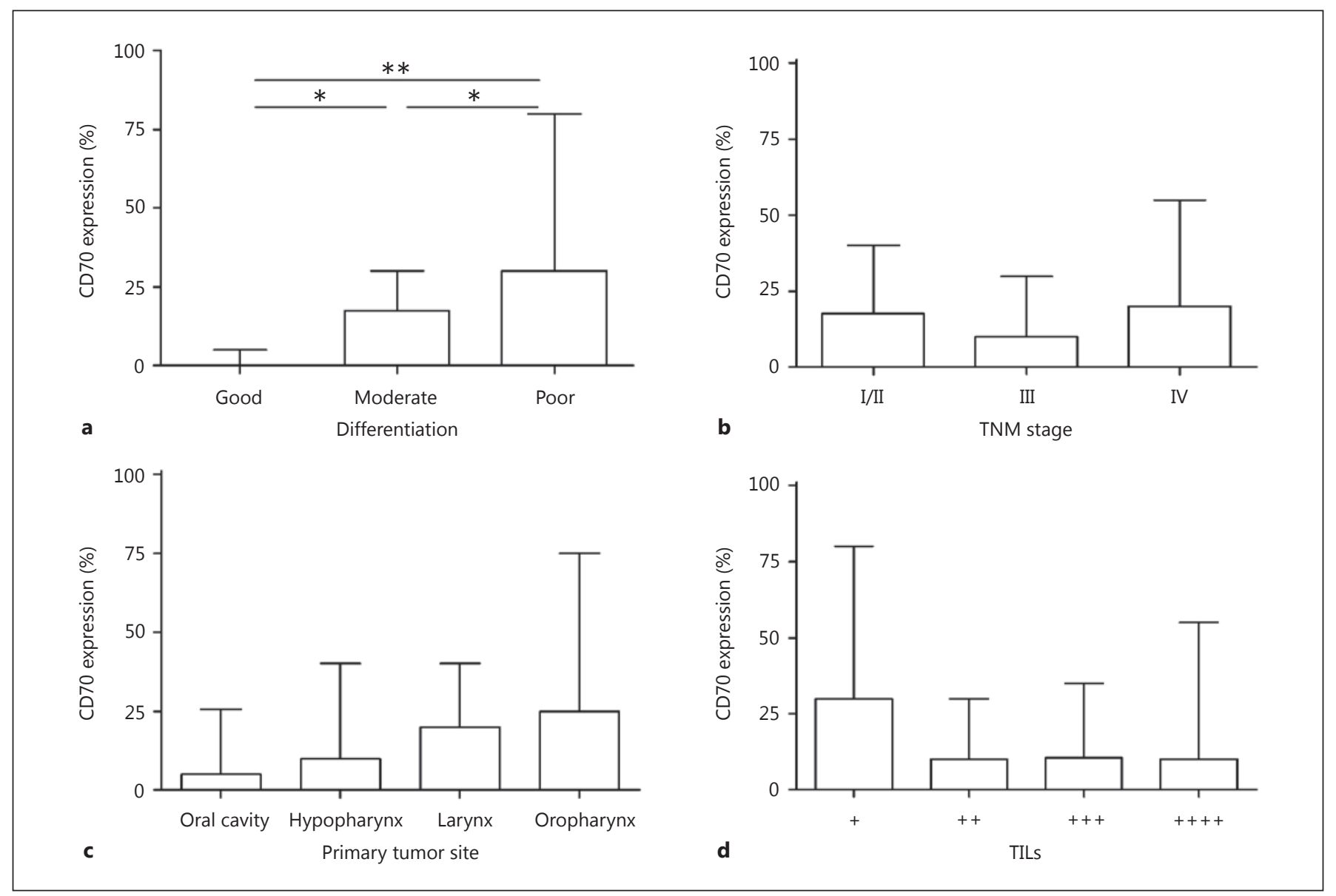

Fig. 3. Association of CD70 expression on tumor cells with clinicopathological variables. Associations are shown for differentiation (a), TNM stage (b), primary tumor site (c) and TILs (d). Statistical significance was calculated using the nonparametric sign test. $\mathrm{p}<0.05$ was statistically significant. ${ }^{*} \mathrm{p}<0.05$; ${ }^{* *} \mathrm{p}<0.01$.

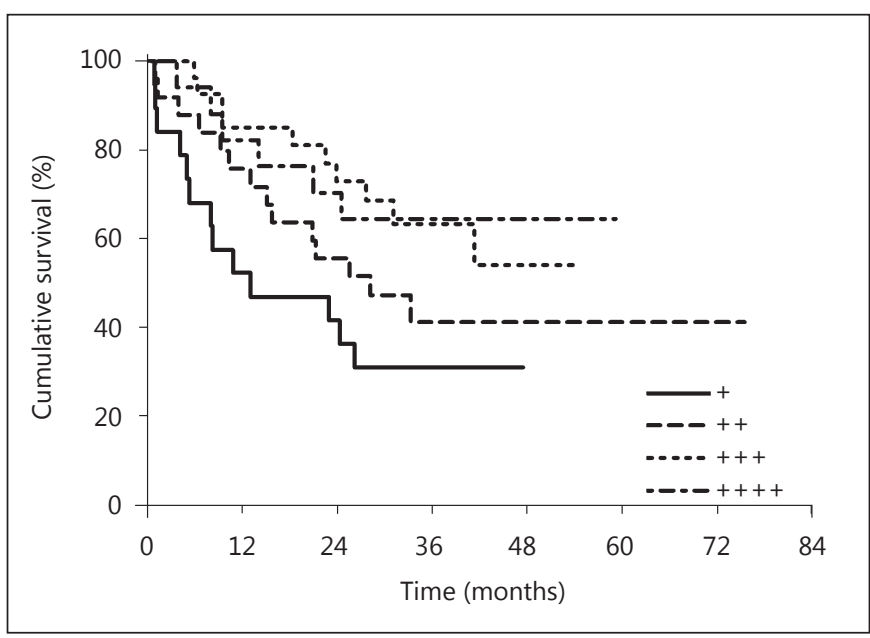

Fig. 4. The density of TILs was significantly associated with OS $(\mathrm{p}=0.042)$.

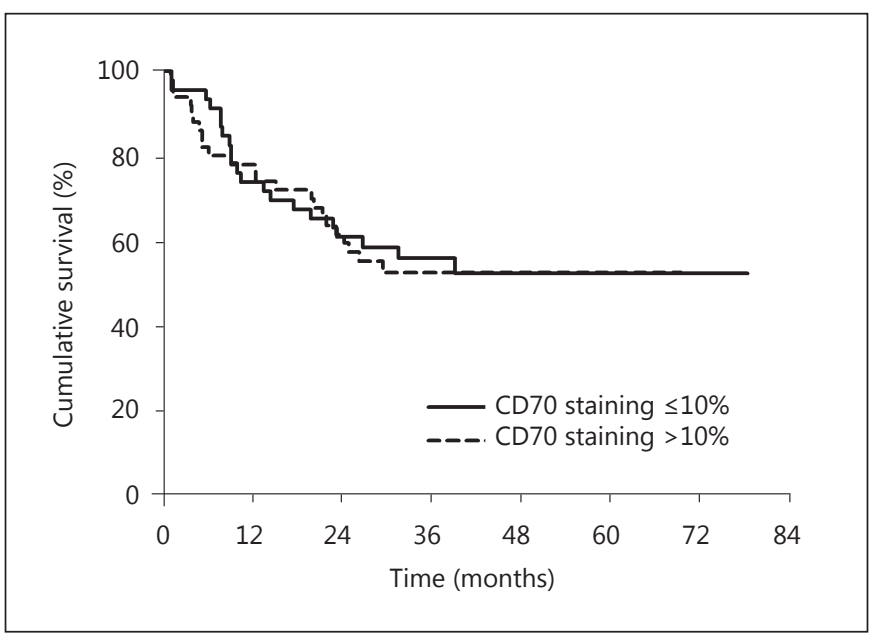

Fig. 5. There was no significant association between CD70 expression and OS when the CD70 expression cut-off was set at $10 \%$. 
for positivity make it impossible to compare these results [8]. Moreover, as demonstrated here, different cellular expression patterns of CD70 can be discerned, i.e. cytoplasmic, membranous, dot-like and (seldom) nuclear. Theoretically, comparable to other antibody-based treatments with immunohistochemical companion diagnostics such as trastuzumab and HER2/Neu in breast and gastric cancer, it is mainly membranous staining that is assumed to be predictive of therapy response, as CD70blocking antibodies operate extracellularly and can only bind the extracellular part of the CD70 molecules. A detailed description and analysis of CD70 IHC in different types of tumor cells is therefore of the utmost importance, enabling us to reveal possible associations between immunohistochemical expression and therapeutic effect.

In agreement with previous reports $[14,15]$, we found that SCCHN patients with tumors densely infiltrated by TILs had a prolonged OS compared to patients with poorly infiltrated tumors. Also, a trend towards less TIL infiltration in SCCHN with higher CD70-expression was observed. Further research on the composition of this TIL infiltrate is needed to elucidate the effect of CD70 expression in tumor cells on these immune cells. In this study, the expression of CD70 on TILs was not taken into account; this probably also contributes to antitumor immune response dynamics.

As $62 \%$ of our samples were endoscopic biopsies, we are aware that sampling error due to tumor heterogeneity may be an issue in the interpretation of these results. However, this option was preferred to excluding these samples from the study. Selecting only resection specimens would inevitably result in a bias towards tumor stage, because only patients with early-stage disease undergo surgical resection.

A second possible drawback in the retrospective design of our study is the heterogeneity of our population. It could be argued that SCCHN is generally considered as a whole in most trials, while recent publications report a heterogeneous molecular and immunological tumor profile of these malignancies at different anatomical locations [16]. However, we deliberately included all tumor sites because, prior to the commencement of our study, it was impossible to predict which types of SCCHN would stain positive for $\mathrm{CD} 70$. In addition, $\mathrm{SCCHN}$ is a rare malignancy, so including different tumor sites enabled the selection of a statistical significant population.

Immunoreactivity for CD70 was not only observed in tumor cells and immune cells as previously described but, interestingly, also in endothelial cells and tumor-associated fibroblasts. This might appear a surprising finding;

however, in recent years, it has become clear that the role of tumor stroma and vasculature is not as straightforward as initially assumed. As endothelial cells closely interact with circulating and transendothelial migrating lymphocytes, they can act as antigen-presenting cells, expressing costimulatory molecules and major histocompatibility complex molecules [17]. Fibroblasts within the tumor stroma have the capacity to modulate the function of tumor-associated T lymphocytes by, e.g. the expression of PD-L1 or the production of immunosuppressive molecules such as TGF- $\beta 1$ or IDO [18]. The limitation of IHC is obviously that protein immunoreactivity does not necessarily reflect protein functionality. Moreover, endothelial cells and fibroblasts show a cytoplasmic staining pattern whereas functional activity, as it is presently known, is expected to come from membranous CD70 molecules. Nevertheless, the meaning and importance of the cellular localization of immunohistochemical CD70 expression remains to be determined.

In summary, our study describes the expression of CD70 in SCCHN. CD70 expression was found in 69\% of SCCHN, displaying a statistical significant association with poor tumor differentiation. These results suggest the involvement of CD70 in SCCHN tumor biology. Further research is needed to elucidate the function of CD70 signaling in the pathophysiology of SCCHN and to investigate the value of anti-CD70 therapy and the use of CD70 $\mathrm{IHC}$ as a predictive marker for anti-CD70 therapy response.

\section{Acknowledgements}

This research was supported by the agency for Innovation by Science and Technology (IWT). Sandrine Aspeslagh was sponsored by the Georges Mathé Translational Research Fellowship.

References

Pathobiology 2016;83:327-333 DOI: $10.1159 / 000446569$
1 Jemal A, Bray F, Center MM, Ferlay J, Ward E, Forman D: Global cancer statistics. CA Cancer J Clin 2011;61:69-90.

2 Gregoire V, Lefebvre JL, Licitra L, Felip E: Squamous cell carcinoma of the head and neck: EHNS-ESMO-ESTRO clinical practice guidelines for diagnosis, treatment and follow-up. Ann Oncol 2010;21(suppl 5):v184v186.

3 http://www.nccn.org/professionals/physician_gls/f_guidelines.asp\#head-and-neck.

4 Wen Y, Grandis JR: Emerging drugs for head and neck cancer. Expert Opin Emerg Drugs 2015;20:313-329. 
5 Wheeler DL, Dunn EF, Harari PM: Understanding resistance to EGFR inhibitors-impact on future treatment strategies. Nat Rev Clin Oncol 2010;7:493-507.

6 Powell SF, Liu SV, Chung ASCH, et al: KEYNOTE-055: a phase II trial of single agent pembrolizumab in patients (pts) with recurrent or metastatic head and neck squamous cell carcinoma (HNSCC) who have failed platinum and cetuximab. ASCO Annu Meet, Chicago, 2015.

7 Borst J, Hendriks J, Xiao Y: CD27 and CD70 in $\mathrm{T}$ cell and B cell activation. Curr Opin Immunol 2005;17:275-281.

8 Jacobs J, Deschoolmeester V, Zwaenepoel K, et al: CD70: an emerging target in cancer immunotherapy. Pharmacol Ther 2015;155: $1-10$.

9 Claus C, Riether C, Schurch C, Matter MS, Hilmenyuk T, Ochsenbein AF: CD27 signaling increases the frequency of regulatory $\mathrm{T}$ cells and promotes tumor growth. Cancer Res 2012;72:3664-3676.
10 Riether C, Schurch C, Ochsenbein AF: Modulating CD27 signaling to treat cancer. Oncoimmunology 2012;1:1604-1606.

11 Coquet JM, Ribot JC, Babala N, et al: Epithelial and dendritic cells in the thymic medulla promote CD4+Foxp3+ regulatory $\mathrm{T}$ cell development via the CD27-CD70 pathway. J Exp Med 2013;210:715-728.

12 Silence K, et al: ARGX-110, a highly potent antibody targeting CD70, eliminates tumors via both enhanced ADCC and immune checkpoint blockade. MAbs 2014;6:523-532.

13 Jacobs J, Zwaenepoel K, Rolfo C, et al: Unlocking the potential of CD70 as a novel immunotherapeutic target for non-small cell lung cancer. Oncotarget 2015;6:13462-13475.
14 Wolf GT, Chepeha DB, Bellile E, et al: Tumor infiltrating lymphocytes (TIL) and prognosis in oral cavity squamous carcinoma: a preliminary study. Oral Oncol 2015;51:90-95.

15 Balermpas P, Michel Y, Wagenblast J, et al: Tumour-infiltrating lymphocytes predict response to definitive chemoradiotherapy in head and neck cancer. Br J Cancer 2014;110: 501-509.

16 Pai SI, Westra WH: Molecular pathology of head and neck cancer: implications for diagnosis, prognosis, and treatment. Annu Rev Pathol 2009;4:49-70.

17 Wheway J, Latham SL, Combes V, Grau GE Endothelial microparticles interact with and support the proliferation of T cells. J Immunol 2014;193:3378-3387.

18 Barnas JL, Simpson-Abelson MR, Yokota SJ, Kelleher RJ, Bankert RB: T cells and stromal fibroblasts in human tumor microenvironments represent potential therapeutic targets. Cancer Microenviron 2010;3:29-47. 